\title{
PECVD Silicon Nitride Diaphragms for Condenser Microphones
}

P. R. SCHEEPER, J. A. VOORTHUYZEN and P. BERGVELD

University of Twente, P.O. Box 217, 7500 AE Enschede (The Netherlands)

\begin{abstract}
The application of plasma-enhanced chemical vapour deposited (PECVD) silicon nitride as a diaphragm material for condenser microphones has been investigated. By means of adjusting the $\mathrm{SiH}_{4} / \mathrm{NH}_{3}$ gas-flow composition, silicon-rich silicon nitride films have been obtained with a relatively low tensile stress. Aluminium can be etched selectively with respect to the silicon nitride films. Using aluminium as a sacrificial layer, $300 \times 300 \mu \mathrm{m}$ silicon nitride diaphragms have been made. Admittance measurements on silicon nitride capacitances have shown that the insulating properties are sufficiently good for application as a microphone diaphragm.
\end{abstract}

\section{Introduction}

Silicon micromachining has become an important tool for the fabrication of many types of sensors and actuators. Recently a capacitive blood-pressure sensor and an electret microphone have been developed, both using silicon micromachining [1].

The first generation of the silicon-based electret microphone consists of an anisotropically etched silicon backplate and a commercially available Mylar diaphragm, which is attached manually per wafer and fixed by means of glue [1]. Disadvantages of using this type of diaphragm are the lack of compatibility with IC technology, wafer-to-wafer irreproducibility and the lack of possibilities of controlling diaphragm stress. Therefore, at present research is directed towards the development of a second generation of the siliconbased microphone, in which diaphragms will

$0925-4005 / 91 / \$ 3.50$ be realized by means of IC-compatible technologies and materials, as for instance presented by Hohm and Hess [2].

In the research described in this paper, sacrificial layer etching has been chosen in favour of wafer bonding, because the use of wafer bonding implies the application of a laborious wafer-alignment step and exposure of the wafers to high electric fields or hightemperature steps.

It has been shown elsewhere that it is preferable that the condenser microphone diaphragm material is insulating for optimization of the signal-to-noise ratio [3]. Silicon nitride is an insulating material whose mechanical properties can be controlled by means of the processing conditions of the chemical vapour deposition process. An advantage of plasma-enhanced with respect to low-pressure chemical vapour deposition is the lower deposition temperature (typically $300{ }^{\circ} \mathrm{C}$ and $800^{\circ} \mathrm{C}$ respectively). The lower deposition temperature of the plasmaenhanced process may be advantageous for the magnitude of the stress in the silicon nitride layer and for the use of other materials with a relatively low decomposition temperature.

The objective of this paper is to investigate if plasma-enhanced chemical vapour deposited (PECVD) silicon nitride can be applied as a condenser microphone diaphragm material. First, the requirements which silicon nitride has to fulfil for the application as a microphone diaphragm material will be discussed. Experimental results concerning the influence of the plasma-enhanced chemical vapour deposition process parameters on the final properties of silicon nitride will be discussed in order to determine which

(C) Elsevier Sequoia/Printed in The Netherlands 
parameter(s) can be used to optimize the silicon nitride. Next, PECVD silicon nitride films will be characterized and finally an example of a silicon nitride diaphragm will be given.

\section{Requirements of Silicon Nitride as a Diaphragm Material}

Most of the thin films being used in IC processing, as deposited on a silicon wafer, exhibit stress which can be tensile or compressive. When etching freestanding micromechanical structures, like beams and diaphragms with clamped edges in a film, the presence of a too large compressive stress will cause buckling [4]. On the contrary, the presence of a tensile stress causes the structures to be stretched. It has been shown that in both cases the sensitivity of a microphone or pressure sensor strongly depends on the stress in the diaphragm [5]. Tensile stress reduces and compressive stress increases the sensitivity. Especially in the design of microphones, the sensitivity is a critical aspect and therefore the realization of structures with compressive stress seems attractive, but on the other hand buckling should be avoided. Therefore it is preferable to realize microphones with a controllably small tensile stress.

The microphone diaphragm will be formed by means of sacrificial layer etching [6] of which the successive processing steps are shown in Fig. 1. First, the sacrificial layer is deposited and patterned, followed by the diaphragm material deposition (a). Small access holes are etched in the diaphragm material (b). The movable diaphragm is formed by sacrificial layer etching via the access holes (c). While the sacrificial layer is etched in the lateral direction for several hundreds of microns, the diaphragm, with a thickness of the order of one micron, should not be attacked by the etchant. With practical dimensions, the ratio between the etch rate of the sacrificial layer and the nitride should be much larger than 1:1000.

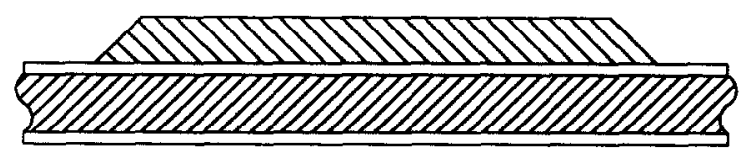

(a)

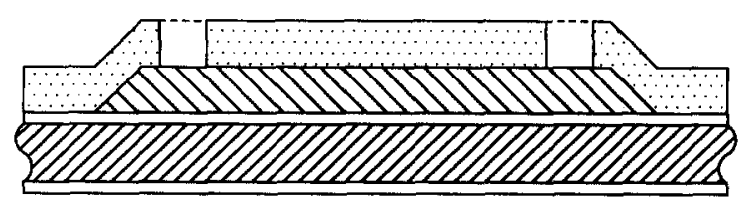

(b)

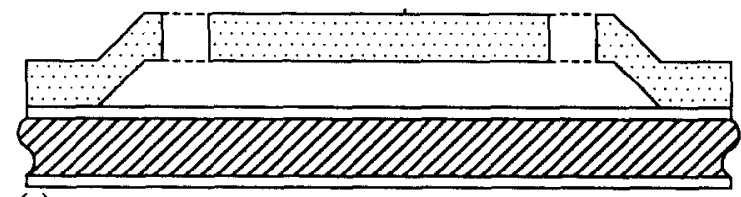

(c)

Fig. 1. Schematic drawing of the diaphragm fabrication process: (a) deposition and patterning of sacrificial layer; (b) deposition of diaphragm material and etching of access holes; (c) etching of the sacrificial layer.

\section{Plasma-enhanced Chemical Vapour Deposition Process Parameters in Relation to Film Stress}

Figure 2 shows a schematic cross section of a parallel-plate plasma-enhanced chemical vapour deposition reactor, in which the lower electrode supports the silicon wafers. The temperature of the lower electrode is kept at a constant value by means of a resistive heating element and a thermocouple. The reactive gases enter the reactor through holes in the upper electrode. Mass-flow controllers keep the flow rate of each gas at a fixed value. The gas pressure in the reactor is controlled by means of a valve between the reactor and the vacuum pump. The plasma is sustained by an r.f. generator, of which the output power can be varied.

Many authors have discussed the influence of the process parameters on the properties of the silicon nitride films produced. It has been found that by varying the substrate temperature, the stress can be varied from compressive, at lower substrate temperatures, to tensile, at higher temperatures [7-9]. The 


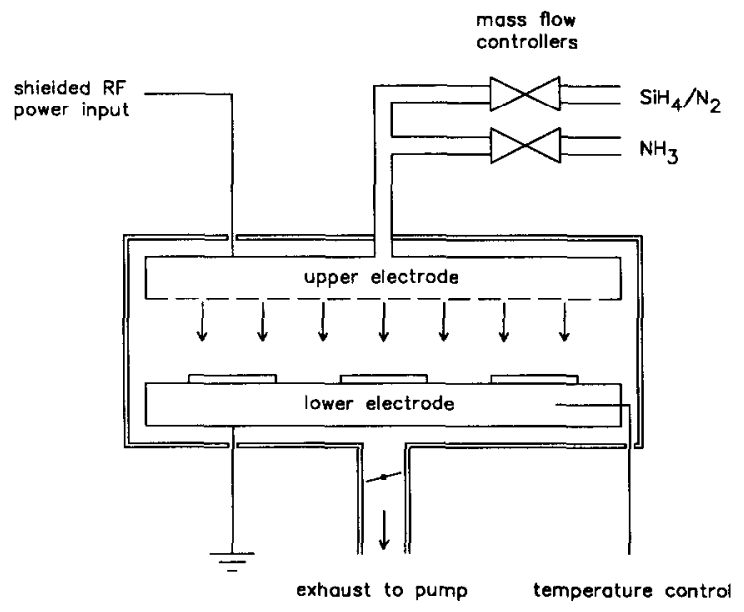

Fig. 2. Schematic cross section of the parallel-plate plasma-enhanced chemical vapour deposition reactor.

temperature at which zero film stress is obtained has been found to vary from $70{ }^{\circ} \mathrm{C}$ [9] to $580^{\circ} \mathrm{C}$ [7], depending on the other experimental conditions, like reactor geometry, composition of reactive gases, gas pressure, plasma frequency, etc. In general, CVD silicon nitride films contain a certain amount of hydrogen, which decreases with increasing substrate temperature $[7,8,10]$. The stress is determined by densification of the film, caused by hydrogen desorption and formation of atomic bonds, which are both temperature-dependent processes, resulting in films with tensile stress for high deposition temperatures. Considering these results, it seems attractive to use the substrate temperature as a tool for optimization of the film stress. However, it has also been found that films deposited at temperatures below $300{ }^{\circ} \mathrm{C}$ are very unstable during additional temperature steps and have a high pinhole density [8]. Both effects are unacceptable for microphone applications, and therefore the deposition temperature should be higher than or equal to $300{ }^{\circ} \mathrm{C}$.

It has been found that the use of low plasma frequencies ( $<4 \mathrm{MHz}$ ) results in films with compressive stress, and the use of high plasma frequencies in films with tensile stress [7]. This is ascribed to the fact that at low frequencies, ions in the plasma are accelerated by the alternating electric field and will reach the substrate surface with high velocity. The kinetic energy of the ions impinging on the growing silicon nitride film is large enough to break atomic bonds, causing the film to expand, resulting in a film with compressive stress. At high frequencies, ion bombardment does not occur because of plasma ion inertness. The film stress is now only determined by hydrogen desorption and formation of atomic bonds and will therefore be tensile. The dependence of the film stress on the plasma frequency has been used to grow low-stress silicon nitride films [9], by applying a plasma that is alternately of high $(13.56 \mathrm{MHz})$ and low $(187.5 \mathrm{kHz})$ frequencies. The intention was to use the ratio of the deposition time of both types of films as a parameter. Unfortunately, it has been found that the film stress changes abruptly from tensile to compressive as a function of the percentage of low frequency.

The effect of the total gas pressure in the reactor on the film stress in silicon nitride has been described for low-frequency deposition only [7]. It has been found that the film stress was always compressive, but that its magnitude decreased as the total gas pressure increased. The gas pressure in the reactor chamber determines the number of collisions between ions and neutral particles in the plasma, and therefore the kinetic energy of ions. As the gas pressure increases, the intensity of ion bombardment decreases, and therefore the compressive stress decreases.

The film stress in silicon nitride has been measured for different gas-flow compositions, using $\mathrm{SiH}_{4} / \mathrm{NH}_{3}$ mixtures diluted with $\mathbf{N}_{2}[9,11]$. For low-frequency deposition $(50 \mathrm{kHz})$, the film stress showed a minimum value, but was always compressive due to ion bombardment [11]. For high-frequency deposition (13.56 MHz), the film stress was compressive for a high $\mathrm{SiH}_{4} / \mathrm{NH}_{3}$ gas-flow ratio and tensile for a low gas-flow ratio [9]. The observed transition from tensile to compressive stress is ascribed to the total hydrogen content of the silicon nitride film and the type of hydrogen bonds that are formed [9].

It can be concluded that stress in PECVD 
silicon nitride films can be controlled between tensile and compressive by means of substrate temperature, plasma frequency and gas-flow composition. The deposition temperature has been chosen as $300{ }^{\circ} \mathrm{C}$, because with the available equipment it was not possible to use temperatures above $300^{\circ} \mathrm{C}$, and temperatures below $300{ }^{\circ} \mathrm{C}$ result in films which are unstable during additional temperature steps and have a high pinhole density. The plasma frequency cannot be varied continuously, because the available equipment could only operate at $187.5 \mathrm{kHz}$ and $13.56 \mathrm{MHz}$. With a plasma that is alternately high- and low-frequency driven, it is still difficult to control the film stress, due to the observed abrupt transition from tensile to compressive stress. Therefore, in the experiments described below, the gas-flow composition has been chosen to control the film stress of silicon nitride layers.

\section{Deposition and Characterization of PECVD Silicon Nitride Films}

The experiments have been carried out with a parallel-plate reactor (Electrotech PF 310). Silicon nitride films with a thickness of $0.5 \mu \mathrm{m}$ have been grown on two inch $\langle 100\rangle$ oriented bare silicon wafers. $\mathrm{SiH}_{4}$ diluted in $\mathrm{N}_{2}(2 \%)$ and $\mathrm{NH}_{3}$ have been used as the reactive gases. Gas pressure, substrate temperature and $\mathrm{SiH}_{4}$ flow have been kept constant at $650 \mathrm{mTorr}, 300^{\circ} \mathrm{C}$ and $2000 \mathrm{sccm}$ respectively. The $\mathrm{NH}_{3}$ flow has been varied in different runs. The r.f. power has been chosen to be $20 \mathrm{~W}$ for high-frequency $(13.56 \mathrm{MHz})$ and $60 \mathrm{~W}$ for low-frequency $(187.5 \mathrm{kHz}) \mathrm{de}-$ position. These conditions were known to yield useful silicon nitride films.

The stress in the silicon nitride films has been measured by using the wafer bending technique [12]. The measured stress as a function of the $\mathrm{NH}_{3}$ flow for high- and lowfrequency deposition is shown in Fig. 3, from which it can be observed that for highfrequency deposition the stress in the silicon nitride can be adjusted as a function of the

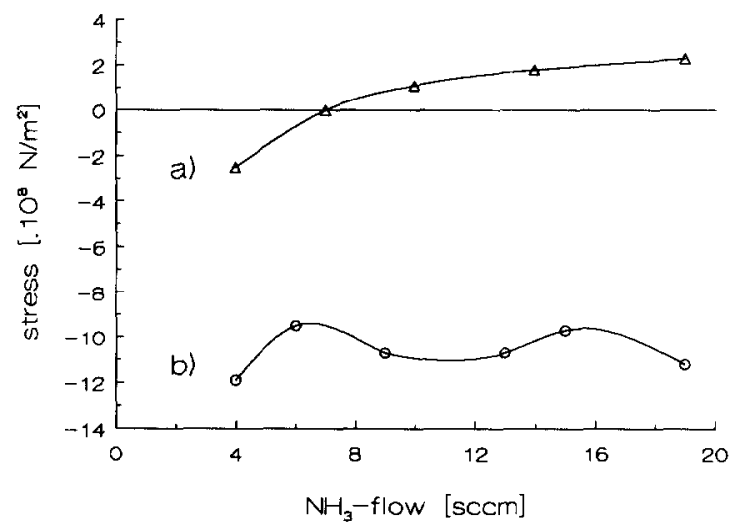

Fig. 3. Measured film stress of silicon nitride as a function of $\mathrm{NH}_{3}$ flow for high and low plasma frequencies. Substrate temperature $300^{\circ} \mathrm{C}$, pressure $650 \mathrm{mTorr}$, $\mathrm{SiH}_{4}$ flow $2000 \mathrm{sccm}$. (a) $13.56 \mathrm{MHz}, 20 \mathrm{~W}$; (b) $187.5 \mathrm{kHz}, 60 \mathrm{~W}$.

$\mathrm{SiH}_{4} / \mathrm{NH}_{3}$ gas-flow composition. Using lowfrequency deposition, films with compressive stress have been obtained, which is in accordance with the literature [11]. Using a $\mathrm{NH}_{3}$ flow of $10 \mathrm{sccm}$ and high-frequency excitation, films with a relatively low tensile stress of $(1.1 \pm 0.2) \times 10^{8} \mathrm{~N} / \mathrm{m}^{2}$ can be grown. The silicon nitride films, grown with a $\mathrm{NH}_{3}$ flow of $7 \mathrm{sccm}$, have a stress too small to be measured with the wafer bending method. Therefore it could not be distinguished whether the stress was tensile or compressive.

The refractive index of silicon nitride is determined by its composition. Figure 4 shows the measured refractive index of silicon nitride films as a function of the $\mathrm{NH}_{3}$ flow. A refractive index larger than 2.0 is typical for silicon-rich films [13], and therefore it may be concluded that the high-frequency deposited films are silicon rich.

The high-frequency deposited silicon-rich silicon nitride films with a tensile stress of $(1.1 \pm 0.2) \times 10^{8} \mathrm{~N} / \mathrm{m}^{2}$ seem to be useful as diaphragm material. Therefore the etch rate in various chemical etchants of this type of film has been determined by measuring the thickness of the film before and after etching. The etch rates in a $\mathrm{HF} / \mathrm{NH}_{4} \mathrm{~F}$ 1:6 solution $\left(27^{\circ} \mathrm{C}\right.$ ), a $33 \mathrm{wt} \% \mathrm{KOH}$ solution (silicon etchant, $70^{\circ} \mathrm{C}$ ) and a $\mathrm{H}_{3} \mathrm{PO}_{4} / \mathrm{CH}_{3} \mathrm{COOH} /$ $\mathrm{HNO}_{3} / \mathrm{H}_{2} \mathrm{O}$ 80:5:5:10 mixture (aluminium 


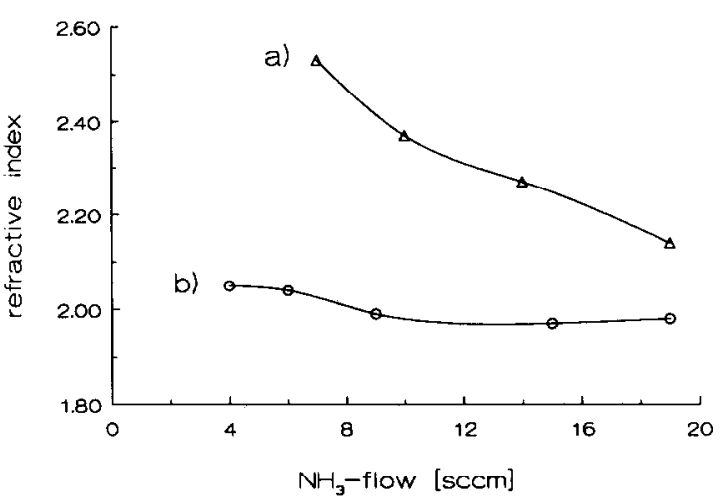

Fig. 4. Measured refractive index of silicon nitride as a function of $\mathrm{NH}_{3}$ flow for high and low plasma frequencies. Temperature $300^{\circ} \mathrm{C}$, pressure $650 \mathrm{mTorr}, \mathrm{SiH}_{4}$ flow $2000 \mathrm{sccm}$. (a) $13.56 \mathrm{MHz}, 20 \mathrm{~W}$; (b) $187.5 \mathrm{kHz}$, $60 \mathrm{~W}$.

etchant, $50^{\circ} \mathrm{C}$ ) have been found to be $840 \mathrm{~nm} / \mathrm{h}, 41.0 \mathrm{~nm} / \mathrm{h}$ and $2.5 \mathrm{~nm} / \mathrm{h}$ respectively. The etch rates of aluminium and silicon, in the above-mentioned etchants, are both $1 \mu \mathrm{m} / \mathrm{min}$, resulting in etch selectivities of $1: 24000$ and $1: 1500$ respectively. The low selectivity of $\mathrm{KOH}$ excludes the use of silicon as a sacrificial layer.

Silicon nitride capacitances have been used for electrical characterization of the silicon nitride films. $600 \times 600 \mu \mathrm{m}$ aluminium electrodes have been defined on top of a $0.5 \mu \mathrm{m}$ silicon nitride film, with the silicon substrate as the opposite electrode. The relative dielec-

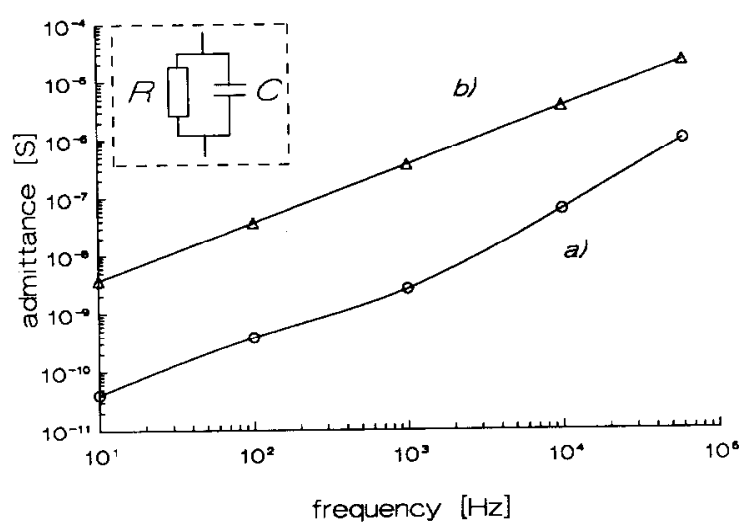

Fig. 5. The measured capacitive and resistive parts of the admittance of a silicon nitride capacitance in the frequency range 10-60 $000 \mathrm{~Hz}$. (a) Resistive part (1/R); (b) capacitive part $(\omega C)$. tric constant, $\epsilon_{\mathrm{r}}$, has been determined from CV measurements and found to be 7.8. The silicon nitride capacitances are considered as a capacitor parallel with a resistor that represents the leakage current through the capacitor. Admittance measurements in the frequency range $10-60000 \mathrm{~Hz}$ have shown that the capacitive part of the admittance is always much bigger than the resistive part, as shown in Fig. 5. The leakage current can be neglected with respect to the total current. Therefore it can be concluded that the insulating properties of silicon nitride are sufficiently good for application as a diaphragm material for condenser microphones.

\section{PECVD Silicon Nitride Diaphragms}

Silicon nitride diaphragms have been realized on $\langle 100\rangle$-oriented p-type silicon wafers provided with $0.2 \mu \mathrm{m}$ thermal oxide. As a sacrificial layer, evaporated aluminium with a thickness of $1 \mu \mathrm{m}$ has been used. $1 \mu \mathrm{m}$ thick silicon nitride has been grown with a tensile stress of $(1.1 \pm 0.2) \times 10^{8} \mathrm{~N} / \mathrm{m}^{2}$ under the experimental conditions shown in Fig. 3. The access holes for sacrificial layer etching, as schematically drawn in Fig. 1, have been formed in the silicon nitride layer by means of reactive ion etching.

Silicon nitride diaphragms of $200 \times 200 \mu \mathrm{m}$

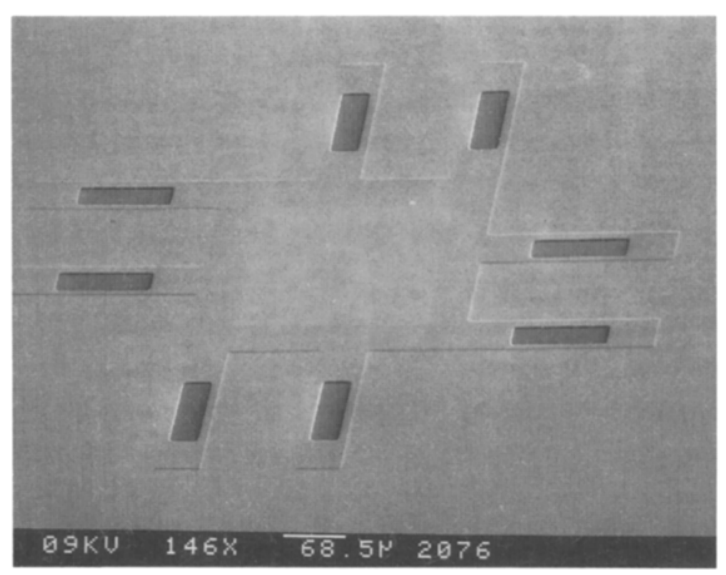

Fig. 6. SEM picture of a $300 \times 300 \mu \mathrm{m}$ silicon nitride diaphragm. 
and $300 \times 300 \mu \mathrm{m}$ have been realized by means of sacrificial layer etching. The times required to complete etching for $200 \times 200 \mu \mathrm{m}$ and $300 \times 300 \mu \mathrm{m}$ diaphragms were about 8 and $24 \mathrm{~h}$ respectively. Figure 6 shows a $300 \times$ $300 \mu \mathrm{m}$ silicon nitride diaphragm. Forcedeflection measurements on a $200 \times 200 \mu \mathrm{m}$ diaphragm with a DEKTAK surface profiler have shown that these diaphragms are not sensitive enough for application as a microphone diaphragm. A rough estimate indicates that the dimensions should be of the order of $1000 \mu \mathrm{m}$.

\section{Conclusions}

PECVD silicon-rich silicon nitride with a relatively low tensile stress of $(1.1 \pm 0.2) \times$ $10^{8} \mathrm{~N} / \mathrm{m}^{2}$ can be grown with a plasma frequency of $13.56 \mathrm{MHz}$ and a $\mathrm{NH}_{3}$ flow of $10 \mathrm{sccm}$. Admittance measurements have shown that the insulating properties of the silicon-rich silicon nitride films are sufficiently good for application as a microphone diaphragm. The etch selectivity of silicon nitride and aluminium has been found to be 1:24 000. The use of silicon as a sacrificial layer is excluded because of the relatively low etch selectivity. Diaphragms can be produced from silicon nitride by means of sacrificial layer etching. The development of larger diaphragms is necessary to produce diaphragms with a suitable sensitivity for a microphone.

\section{References}

1 J. A. Voorthuyzen, P. Bergveld and A. J. Sprenkels, Semiconductor-based electret sensors for sound and pressure, IEEE Trans. Electr. Insul., 24 (1989) 267276.
2 D. Hohm and G. Hess, A subminiature condenser microphone with silicon nitride membrane and silicon backplate, J. Acuust. Soc. Am., 85 (1989) 476480.

3 A. J. Sprenkels, A silicon subminiature electret microphone, Ph.D. Thesis, University of Twente, The Netherlands, 1989, pp. 98-102.

4 H. Guckel, T. Randazzo and D. W. Burns, A simplc technique for the determination of mechanical strain in thin films with applications to polysilicon, J. Appl. Phys., 57 (1985) 1671-1675.

$5 \mathrm{~J}$. A. Voorthuyzen, The influence of tensile forces on the deflection of circular diaphragms in pressure sensors, Sensors and Actuators, 6 (1984) $201-$ 213.

6 R. T. Howe and R. S. Muller, Polycrystalline and amorphous silicon micromechanical beams: annealing and mechanical properties, Sensors and Actuators, 5 (1983) 447-454.

7 W. A. P. Claassen, W. G. J. N. Valkenburg, M. F. C. Willemsen and W. M. Van der Wijgert, Influence of deposition temperature, gas pressure, gas phase composition, and RF frequency on composition and mechanical stress of plasma silicon nitride layers, $J$. Electrochem. Soc., 132 (1985) 893-898.

$8 \mathrm{~K}$. Allaert, A. Van Calster, H. Loos and A. Lequesne, A comparison between silicon nitride films made by PCVD of $\mathrm{N}_{2} \mathrm{SiH}_{4} / \mathrm{Ar}$ and $\mathrm{N}_{2} \mathrm{SiH}_{4}$ / He, J. Electrochem. Soc., 132 (1985) 1763-1766.

9 A. Kiermasz, J. Bhardwaj and A. McQuarrie, A novel technique for controlling stress in PECVD silicon nitride layers, Vide Couches Minces, (1987) 237-239.

10 H. Dun, P. Pan, F. R. White and R. W. Douse, Mechanisms of plasma-enhanced silicon nitride deposition using $\mathrm{SiH}_{4} / \mathrm{N}_{2}$ mixture, J. Electrochem. Soc., 128 (1981) 1555-1563.

11 G. M. Samuelson and K. M. Mar, The correlations between physical and electrical properties of PECVD SiN with their composition ratios, J. Electrochem. Soc., 129 (1982) 1773-1778.

12 R. J. Jaccodine and W. A. Schlegel, Measurement of strains at $\mathrm{Si}-\mathrm{SiO}_{2}$ interface, J. Appl. Phys., 37 (1966) 2429-2434.

13 S. V. Nguyen and S. Fridmann, Plasma deposition and characterization of thin silicon-rich silicon nitride films, J. Electrochem. Soc., 134 (1987) 2324 2329. 